\title{
3 Research Soure \\ Predictors to HIV testing among youth women in Ethiopia: 2016 Ethiopian Demographic and Health Survey
}

Gedefaw Diress ( $\square$ gedefawdiress@gmail.com )

Woldia University

Mohammed Ahmed

Woldia University

Seteamlak Adane

Woldia University

Melese Linger

Woldia University

Birhan Aleminew

Woldia University

Research article

Keywords: HIV testing, youth women, Ethiopia Demographic and health survey

Posted Date: April 17th, 2019

DOl: https://doi.org/10.21203/rs.2.9217/v1

License: (9) This work is licensed under a Creative Commons Attribution 4.0 International License.

Read Full License 


\section{Abstract}

Background HIV testing is the critical first step in identifying and linking HIV infected people to the treatment cascade and it also provides an important opportunity to reinforce HIV prevention among the negatives. The aim of this study was examine factors associated with HIV testing among youth women. Methods A community-based cross-sectional study design was used and a nationally representative secondary data analysis was done on the 2016 Ethiopian Demographic and Health Survey(EDHS). A total of 6401 youth women were eligible in the study. The data were analyzed by SPSS version 20. Frequencies and weighted percentage of the variables were calculated. Chi-square tests and logistic regression models were used to assess predictors of HIV testing. Multivariate logistic regression analysis was conducted to control confounders and to identify the independent contribution of each variable to the outcome variable. Result A total of 6401 youth women aged 15 to 24 years of age were included. Only $37.7 \%$ (95\% Cl:(33.6-39.1\%)) of participants were ever tested for HIV in their life. In the final multivariable model age, marital status, level of educational, media access, number of sexual partner, STIs in Past 12 months and comprehensive knowledge to HIV, were significantly associated with ever been tested for HIV. Youth women who were in the age group between 20 to 24 (AOR=2.18; 95Cl:(1.800-2.652), who were married (AOR=4.70; 95\% Cl:(3.674-6.008)), were divorced (AOR=6.16; 95\% Cl (3.976-9.541)), who had no access to media $(A O R=0.69 ; 95 \mathrm{Cl} \%$ : $(0.540-0.870)$ ), who had no comprehensive HIV knowledge (AOR = $0.68 ; 65 \% \mathrm{Cl}:(0.530-0.861))$ and having one or more sexual partners (AOR=2.48; 95\% Cl:(1.350-4.551)) were significantly associated with ever been tested for HIV.

\section{Background}

Worldwide, the number of young people within the age of 15 to 24 is increasing in a regularly state and about 3.9 million Young's (15-24) were living with HIV in 2014 and the number of infected adolescents risen by $30 \%$ between 2005 and 2016 with more infected women's(1) and approximately $50 \%$ of all new HIV infections(2). In Sub-Saharan Africa persons aged 10-24 years constitute the largest proportion of the population (3). In Ethiopia, according to population projections for 2017, adolescents and youth aged 1024 account for one third of the population $(31,426,691)$ and about half of them $(15,485,880)$ are adolescent girls and young women aged 10-24years(4) but several are unaware of their HIV status (5) .

In Ethiopia, the nationwide HIV prevalence in 2017 was 1.16\% (6) and there were an estimated 613,000 people living with HIV, of whom 62 percent was female, in 2017(4). HIV infection is high among Ethiopian youth but only $76.9 \%$ of HIV-positive females who are in reproductive age group knew their HIV status(6). In Ethiopia youth are among the key populations disproportionately affected by HIV/AIDS(7). In addition, Ethiopia is one of the selected countries for Global HIV Prevention Coalition due to highest numbers of new HIV infections(8).

In 2014, the Joint United Nations Programme on HIV/AIDS and partners established goals(90-90-90 targets) to identify $90 \%$ of the people living with HIV, offer treatment to $90 \%$ of those detected with HIV, 
and attain viral suppression among $90 \%$ of those on treatment by 2020 to help end the AIDS epidemic by 2030(9).

So, HIV testing is the critical first step in identifying and linking HIV infected people to the treatment cascade and it also provides an important chance to strengthen HIV prevention among the negatives. One of the main objectives in HIV testing and counseling is to identify and link HIV positive persons to care and treatment services and HIV negative people to prevention services(10).

Timely testing and access to treatment is related with a reduced likelihood of forward transmission (11) better response to antiretroviral treatment (ART) and reduced mortality and morbidity(12). For this reason, starting from September 2015, World Health Organization's (WHO) recommended that all individuals be treated as soon as possible after HIV infection and diagnosis(13). In Ethiopia, there are two kinds of models: health facility based HIV testing and counseling model and community based HIV testing and counseling model(10).

Based on study done in American adults, $50 \%$ of participants reported past-year HIV testing(14) and report in south Africa stated that among sexually experienced youth, $32.7 \%$ of females and $17.7 \%$ of males reported having been tested for HIV(15). Up-to-date study based on Demographic and Health Survey (DHS) data from 16 Sub-Saharan African countries assessed that merely $54 \%$ of people living with HIV (range across countries $26 \%$ to $84 \%$ ) were aware of their status, contributing to delays in care enrollment and ART initiation(16).

Based on results from Sierra Leone Demographic Health Survey, only $12 \%$ of the participants had ever taken a test for HIV(17) and 61\% of women ever been tested for HIV from Zimbabwe DHS(18)

According to study in developed countries age, lifetime STI testing and diagnosis, peer norms in support of HIV testing, access to HIV testing running out of money for basic necessities in the past year, alcohol use, access to general medical care, income and recent substance use, fear of contracted HIV through sexual behavior and health care recommendations $(14,19)$ were factors associated with HIV testing.

In Africa, barriers to HIV testing consist of lack of awareness of available services, low perception of personal risk, fear of negative consequences associated with a positive test result (including stigma), concerns about confidentiality, financial burden of testing, and lack of HIV/AIDS knowledge, pregnancy status, conversation about HIV/AIDS and residence, number of sexual partners, perception about benefit for HIV testing (20-23).

Residence, number of under five children, education status, age at first sex, used condom at last sex, and know that HIV can be reduced by using condom were associated with HIV testing(17). Age, educational status, wealth index, marital status, ANC follow up in the 5 years, number of sexual partners, consistent condom use in last 12 months and ever had paid for sex in Zimbabwe(18) gender, age, age at sexual debut, and comprehensive knowledge of HIV in Sub-Saharan Africa (24) were identified factors that affect HIV testing 
In Ethiopia, only 24 percent of women aged 15-24 years have comprehensive knowledge of HIV so significant proportion of young women (40\%) have sex before age of 18 years(8) which be one of reason for low proportion of HIV testing.

To address high unmet need for HIV care \& treatment and accelerate progress towards the UNAIDS 90-9090 targets in Ethiopia, nationwide information regarding HIV testing is needed. But, research on women especially youth concerning HIV testing and associated factors was limited in Ethiopia.; So, this paper was used to examine the prevalence and factors associated with HIV testing among youth women to inform future policies, gender appropriate interventions, and HIV testing initiatives.

\section{Methods}

\section{Study design}

A community-based cross-sectional study design was used, and a nationally representative secondary data analysis was done on the 2016 EDHS. The samples were selected using a two-stage stratified cluster sampling technique.

\section{Data source}

The source of data for this study is EDHS 2016 which was collected from January 18, 2016, to June 27, 2016.

\section{Description about 2016 EDHS}

The sampling frame used for the 2016 EDHS is the Ethiopia Population and Housing Census (PHC), which was conducted in 2007 by the Ethiopia Central Statistical Agency. The census frame is a complete list of 84,915 enumeration areas (EAs) created for the 2007 PHC. An EA is a geographic area covering on average 181 households. Administratively, Ethiopia is divided into nine geographical regions and two administrative cities. The 2016 EDHS sample was stratified and selected in two stages. Each region was stratified into urban and rural areas, yielding 21 sampling strata. Samples of EAs were selected independently in each stratum in two stages.

In the first stage, a total of 645 EAs (202 in urban areas and 443 in rural areas) were selected with probability proportional to EA size (based on the $2007 \mathrm{PHC}$ ) and with independent selection in each sampling stratum.

In the second stage of selection, a fixed number of 28 households per cluster were selected with an equal probability systematic selection from the newly created household listing. All women age 15-49 and all men age 15-59 who were either permanent residents of the selected households or visitors who stayed in the household the night before the survey were eligible to be interviewed

\section{Source Population}


Ethiopian youth women (15-24)

\section{Study population}

Ethiopian youth women who respond to outcome variable (previous HIV test yes/no)

Inclusion criteria: youth women who respond to outcome variable

\section{Study Variables Description}

In this study the outcome variable, self-reported previous HIV testing (yes/no) was defined as having accessed HIV testing services at least once in their lifetime prior to this survey.

Independent variables were Residence, Educational status, Wealth index, Sex of head of the household, Age, Access to media, Internet use, Substance uses, self-reported history of sexually transmitted infections, pregnancy status, number of under five children, comprehensive knowledge to HIV, consistent condom use in the past 12 months and number of lifetime sexual partners.

\section{Operational definition}

Comprehensive knowledge: - correctly responded all of the following 5 questions

- Consistent use of condoms during sexual intercourse can reduce the chance of getting HIV

- Having just one uninfected faithful partner can reduce the chance of getting HIV

- A healthy-looking person can have HIV

Rejecting the two most common local misconceptions about transmission or prevention of HIV.

- HIV can be transmitted by mosquito bites and

- A person can become infected by sharing food with a person who has HIV.

Youth: age in years between 15-24

Access to media: those women who use either newspaper, television or radio

Data extraction and analysis: women data from EDHS 2016 was extracted. The data were analyzed by SPSS version 20. Frequencies, and weighted percentage of study variables were calculated. Crosstabulation analyses between HIV testing, sociodemographic, and other variables was performed. The variables that had statistically significant associations with previous HIV testing (as determined using Rao-Scott's $\chi 2, p<.05)$ in the bivariate analyses were all used in the final multivariable logistic regression model to control confounders and to identify the independent contribution of each variable to the outcome variable. The statistical tests were reported based on $95 \%$ confidence interval in the final model. 


\section{Ethical consideration}

Ethical clearance for the survey (EDHS 2016) was provided by the Federal Democratic Republic of

Ethiopia Ministry of Science and Technology and the Institutional Review Board of ICF International. Respondents were informed about the survey and consent was taken for their participation. Voluntary participation was ensured during interviews. The researchers have received the survey data from Measure DHS and then the researchers of this study have maintained the confidentiality of the data.

\section{Results}

\section{Socio demographic and health related characteristics of participants}

A total of 6401 youth women aged 15 to 24 years of age were interviewed. Around $55 \%$ of participants were in the age group between $15 \& 19$ years and $76.1 \%$ were rural resident. Orthodox and Muslim religion constitute $43 \%$ and $30.7 \%$ of religion respectively. Majority $(57 \%)$ were single/never in union and the proportion of youth women with no formal education was $20 \%$. A small proportion (8.6\%) of youths ever chewed chat and almost half of participants had access to media. Only $24.3 \%$ of young women had comprehensive knowledge to HIV and only $7.5 \%$ of youth used internet. Only small proportion $(37.7 \%)$ of participants were ever tested for HIV in their life (see table 1.)

\section{Predictors for HIV testing}

During bivariate analysis model age, place of residence, marital status, level of educational, media access and comprehensive knowledge to HIV were significantly associated with HIV testing. In bivariate analysis women in the age group 20-24 had higher odds of experiencing HIV testing than the 15-19 age groups $(C O R=3.33, p=0.000)$. Similarly, those who are from urban residence had higher odds of HIV testing $(\mathrm{COR}=2.55, \mathrm{p}=0.012)$ when compare to rural. Those youth women who are married, divorce and widowed had higher odds of HIV testing ( $p$-value $=0.001$ ) than single. Educated women (primary, secondary and higher level) were more likely to practice HIV testing than non-educated one. Regarding media access, women who had no access to media were less likely to be tested for HIV (COR=0.46(95\% Cl:0.380-0.549)) than who had media access. Similarly, youth who had no comprehensive knowledge to HIV were less likely to be ever tested for HIV when compare to their counterpart.

In the final multivariable model, age, marital status, level of educational, media access, number of sexual partner, STIs in Past 12 months and comprehensive knowledge to HIV, were significantly associated with ever been tested for HIV.

Those youth women who were in the age group between 20 to 24 were 2.18 times more likely to be ever tested for HIV as compared with who were in the age group 15-19 years. The odds of ever tested for HIV were 4.7 times (AOR=4.70; $95 \% \mathrm{Cl}$ :(3.674-6.008)) higher among those who were married, 6.2 times $(A O R=6.16 ; 95 \% \mathrm{Cl}(3.976-9.541)$ ) higher among those who were divorced than never in union/single. But, being widowed is not statistically significant associated with the dependent variable. 
Those women who had no access to media had $31 \%(A O R=0.69 ; 95 \mathrm{Cl} \%$ : (0.540-0.870)) lower odds of HIV testing when compare to those who had access to media. Similarly, women who had no comprehensive HIV knowledge had $32 \%(A O R=0.68 ; 65 \% \mathrm{Cl}$ : $(0.530-0.861)))$ lower odds of HIV testing when compare to those who had comprehensive HIV knowledge. Having one or more sexual partners other than spouse was associated with an increase odd of having been tested (AOR=2.48; $95 \%$ Cl:(1.3504.551)) (table 2.)

\section{Discussion}

HIV/AIDS is the key problem and a great challenge in in Sub-Saharan Africa today. In Ethiopia youth are among the key populations extremely affected by HIV/AIDS. In spite of the scale up of HIV Testing and Counselling(HTC) services in Ethiopia, this study shows that HIV testing practice among youth women are low.

In this study, only $37.7 \%$ youth women had ever been tested for HIV. This is consistent with study conducted in South Africa which is $32.7 \%$ (15). But greatly higher than a study conducted in Sierra Leone which was only $12 \%(17)$. In contrast to this, it is much lower than a study conducted in Zimbabwe (61\% of women ever been tested for HIV)(18) and other Sub-Saharan African countries (16).

Women who were in the age group between 20 to 24 were more likely to be ever tested for HIV as compared with who were in the age group 15-19 years. This might be due to older women have more lifetime exposure to the possibility of being tested and may have accessed HIV testing during other maternity service like antenatal follow up care. This is supported with studies done in different parts of Africa $(17,23,24)$ and in Haiti $(25)$. But, this is in disagreeing to the finding from New York city (14) which stated that older age was associated with a decrease in the odds of testing for HIV. This difference might be due to age difference in study population.

This study revealed that youth women who were married were more likely to be ever tested for HIV than single or never in union. This might be due to married women were more probable to know their status before marriage. In addition, married women might be tested for HIV as part of antenatal care during pregnancy. This finding is supported by a study conducted in Zimbabwe and rural western Uganda (18, 26) but there was no association between marital status and HIV testing in study done in south Africa (23).

The likelihood of having been tested increased in women with highest educational level when compared with women without any formal education. As educational level increase the probability being ever tested for HIV was increased because prolonged exposure to HIV prevention messages that are more readily available in the school system than in the community $(23,27)$.

Regarding to wealth index, being poorest were barrier for HIV testing in youth women. This might be due to lack of money because of high payment for HIV testing in private health institution. Even though the test was free of charge in all governmental health institution in Ethiopia, youth prefer private health 
institution for their confidentiality. This finding that is consistent with research conducted in Mozambique (28) and in Haiti (25) in which lack of financial resources was barriers to HIV testing.

HIV testing was higher among women who had one or more sexual partners other than spouse. Women who have multiple partners are more likely to test for HIV because they thought that they had gotten HIV from risky sexual behavior. Furthermore, male partners may also enforce or initiate them to be tested. It also supported by other studies $(18,23,25,29)$.

This study revealed higher levels of HIV testing among youth with comprehensive HIV knowledge were more likely to be ever tested for HIV. This implies creating awareness about HIV in the communities leads to an increase in the uptake of HIV testing. In opposite to this, there was no association in previous study conducted in Zimbabwe(18).

The odds of ever having been tested was higher among women reporting to have STIs in the last 12 months (AOR=1.50; $95 \% \mathrm{Cl}$ : (0.379-5.906) than their counter part. This finding might be due to their risky sexual behavior might rise their need for testing and health care provider might initiate them to be tested since STI patients were selected priority population group for HIV testing in Ethiopia.

\section{Conclusion}

Since, HIV infection is high among Ethiopian youth, HIV testing and counselling in this group is a public health priority. But, HIV testing practice among youth women is low (37.7\%) in our study. To improve uptake HIV test there should be ongoing efforts like improve availability of youth-friendly service, builds HIV/AIDS clubs at secondary schools and higher education and target based testing.

HIV testing was lower in younger people, and this sounds for policymakers to build up approaches targeted at improving HIV testing among these groups (15-19 years) and strengthen earlier key national documents that emphasize this age group like HIV/AIDS Strategic Plan 2015-2020.

Policymakers should investigate tactics targeted at scaling up HIV testing in the lower level-income and non-educated women since higher educational level was positively associated with HIV ever tested and decreased proportions of tested among those with low wealth status. Promotion of education may be a potential means to increase HIV testing in Ethiopia.

\section{Strength of study}

A nationally representative sample survey which used a validated questionnaire.

\section{Limitation of study}

- It is a cross-sectional survey; causality cannot be determined from the findings.

- There may social desirability bias in participants' responses. 


\section{Declaration}

Ethical approval and consent to participate

Not applicable

Consent for publication

Not applicable

Availability of data

The data can be available from corresponding author and DHS data base

Competing interest

The authors declare that they have no competing interests

Funding

Not applicable

Acknowledgment

None

\section{Authors' information}

GD: BSC, MPH in Epidemiology; working in Woldia University, Faculty of Health Sciences, Woldia, Ethiopia. MA: BSC, MPH in Epidemiology; working in Woldia University, Faculty of Health Sciences, Woldia, Ethiopia.SA: BSC, MPH in Reproductive health; working in Woldia University, Faculty of Health Sciences, Woldia, Ethiopia. ML: BSC, MPH in Nutrition; working in Woldia University, Faculty of Health Sciences, Woldia, Ethiopia. BA: BSC, MSC in Medical Microbiology, working in Woldia University, Faculty of Health Sciences, Woldia, Ethiopia

\section{List Of Abbreviations}

AOR-Adjusted Odds Ratio, COR-Crude Odds Ratio, EDHS-Ethiopia Demographic Health Science, HIVHuman Immune-Deficiency Virus, SPSS-Statistical Package for Social Science

\section{Reference}

1. WHO. Adapting and Implimenting new recommendaation on HIV patient monitoring,2017. 
2. All in to \#End Adolescent AIDS. USAIDS website

http://www.unaids.org/sites/default/files/media_asset/20150217_ALL_IN_brochure.pdf Accessed 2019.

3. UNICEF. Progress for Children: A report card on adolescents. 2012.

4. Central Statistical Agency. Population Projections for Ethiopia 2007-2037. 2013.

5. Lancaster KE, Hetrick A, Jaquet A, Adedimeji A, Atwoli L, Colby DJ, et al. Substance use and universal access to HIV testing and treatment in sub-Saharan Africa: implications and research priorities. J Virus Erad. 2018;4(Suppl 2):26-32.

6. ETHIOPIA POPULATION-BASED HIV IMPACT ASSESSMENT(EPHIA) 2017-2018.

7. Central StatisticalAgency (CSA)[Ethiopia], ICF. Ethiopia Demographic and Health Survey(EDHS). 2016.

8. HIV Prevention in Ethiopia National Road Map 2018 - 2020.

9. UNAIDS. 90-90-90: on the right track towards the global target. Geneva: Switzerland; 2016.

10. National guidelines for comprhensive HIV prevention care and treatment,Ethiopia, MOH. 2017.

11. Fox J, White PJ, Macdonald N, et al. Reductions in HIV transmission risk behaviour following diagnosis of primary HIV infection: a cohort of high-risk men who have sex with men. HIV Med. 2009;10(7):5-8.

12. Egger $M$, May M, Chene $G$, et al. Prognosis of HIV-1-infected patients starting highly active antiretroviral therapy: a collaborative analysis of prospective studies. Lancet. 2002;360(9327):119-29.

13. Yotebieng $M$, et al. Research priorities to inform "Treat All" policy implementation for people living with HIV in sub-Saharan Africa: a consensus statement from the International epidemiology Databases to Evaluate AIDS (IeDEA). Journal of the International AIDS Society. 2018.

14. Gwadz M, Cleland CM, Kutnick A, Leonard NR, Ritchie AS, Lynch L, et al. Factors Associated with Recent HIV Testing among Heterosexuals at High Risk for HIV Infection in New York City. Front Public Health 2016;4(76).

15. Meehan SA, Sloot R, Draper HR, Naidoo P, Burger R, Beyers N. Factors associated with linkage to HIV care and TB treatment at community-based HIV testing services in Cape Town, South Africa. PLoS One. 2018;13(4):e0195208.

16. Staveteig S, Croft TN, Kampa KT, Head SK. Reaching the 'first 90': gaps in coverage of HIV testing among people living with HIV in 16 African countries. PLoS One. 2017;12(10):4-9.

17. Brima N, Burns F, Fakoya I, Kargbo B, Conteh S, Copas A. Factors Associated with HIV Prevalence and HIV Testing in Sierra Leone: Findings from the 2008 Demographic Health Survey. PLoS One. 
2015;10(10):e0137055.

18. Takarinda KC, Madyira LK, Mhangara M, Makaza V, Maphosa-Mutsaka M, Rusakaniko S, et al. Factors Associated with Ever Being HIV-Tested in Zimbabwe: An Extended Analysis of the Zimbabwe Demographic and Health Survey (2010-2011). PLoS One. 2016;11(1):e0147828.

19. Debra A. Murphy, Rick Mitchell, Sten H. Vermund, Futterma D. Factors Associated With HIV Testing Among HIV-Positive and HIV-Negative High-Risk Adolescents. Pediatrics. 2002.

20. Musheke M, Ntalasha H, Gari S, Mckenzie O, Bond V, Martin-Hilber A, et al. A systematic review of qualitative findings on factors enabling and deterring uptake of HIV testing in Sub-Saharan Africa. BMC Public Health. 2013.

21. Oginni A, Obianwu O, Adebajo S. Socio-demographic Factors Associated with Uptake of HIV Counseling and Testing (HCT) among Nigerian Youth. . AIDS Research And Human Retroviruses. 2014;30.

22. Schwarcz S, Richards TA, Frank H, Wenzel C, Hsu LC, Chin CS, et al. Identifying barriers to HIV testing: personal and contextual factors associated with late HIV testing. AIDS Care 2011;23(7):8-12.

23. Lucy Chimoyi, et al. HIV-related knowledge, perceptions, attitudes, and utilisation of HIV counselling and testing: a venue-based intercept commuter population survey in the inner city of Johannesburg, South Africa. Global health, science and practice. 2015.

24. Ibitola O. Asaolu, Jayleen K. Gunn, Katherine E. Center, Mary P. Koss, Julietl. Iwelunmor, John E. Ehiri. Predictors of HIV Testing among Youth in Sub-SaharanAfrica: A Cross-SectionalStudy. PLoS ONE. 2016;11(10):2-14.

25. Donaldson F. Conserve, Juliet Iwelunmor, Guy-Lucien Whembolua, Yewande Sofolahan-Oladeinde, Michelle Teti, Pamela J. Surkan P. Factors Associated With HIV Testing Among Men in Haiti: Results From the 2012 Demographic and Health Survey. American Journal of Men's Health. 2017;11(5).

26. Bwambale FM, Ssali SN, Byaruhanga S, Kalyango JN, Karamagi CA. Voluntary HIV counselling and testing among men in rural western Uganda: implications for HIV prevention. BMC Public Health. 2008;8.

27. Hargreaves J, et al. The association between school attendance, HIV infection and sexual behaviour among young people in rural South Africa. Journal of Epidemiology \& Community Health. 2008;62.

28. Agha S. Factors associated with HIV testing and condom use in Mozambique: Implications for programs. Reproductive Health. 2012.

29. Mhlongo S, Dietrich J, Otwombe KN, Robertson G, Coates TJ, Gray G. Factors associated with not testing for HIV and consistent condom use among men in Soweto, South Africa. PLoS One. 2013;8(5).

\section{Tables}


Table 1. Background information of participants $(\mathrm{N}=6401)$ 
Variables

Age

15-19

20-24

Residence

Urban

Rural

Religion

Orthodox

Muslim

Protestant

Catholic

Traditional

Others

\section{Marital status}

Never union/single

Married

Divorce

Widowed

Living with partner

No longer living together

\section{Educational status}

No education

Primary

Secondary

Higher

Wealth index

Poorest
Total n(\%) Ever tested for

HIV

p-

value

No(n=3741) Yes(n=2660)

0.000

\begin{tabular}{lll}
\hline $3498(55.0)$ & $2465(66.0)$ & $1033(36.9)$ \\
\hline $2903(45.0)$ & $1276(34.0)$ & $1627(63.1)$
\end{tabular}

0.000

2340(23.9) 1019(17.3)

1321(34.8)

4061(76.1) 2722(82.7)

$1339(65.2)$

0.000

2613(43.0) 1250(35.7) 1363(54.9)

2569(30.7) 1748(35.5) 821(22.6)

1135(24.2) $\quad 680(26.3) \quad 455(20.8)$

$\begin{array}{lll}37(0.9) & 23(0.9) & 14(0.9)\end{array}$

24(0.9) 20(1.1) 4(0.6)

23(0.4) 20(0.6)

$3(0.1)$

0.001

3622(57.0) 2442(66.6) 1180(41.1)

2366(36.2) 1133(28.6) 1233(48.9)

245(3.7) 109(2.7) 136(5.5)

12(0.2) $9(0.3) \quad 3(0.1)$

$81(1.2) \quad 19(0.7) \quad 62(2.1)$

$75(1.7) \quad 29(1.3) \quad 46(2.4)$

0.024

\begin{tabular}{lll}
\hline $1408(20.0)$ & $1098(24.3)$ & $310(12.9)$ \\
\hline $3104(54.3)$ & $1910(57.4)$ & $1194(49.0)$ \\
\hline $1361(19.3)$ & $598(15.3)$ & $763(25.9)$ \\
\hline $528(6.4)$ & $135(3.0)$ & $393(12.2)$
\end{tabular}

0.000

1476(15.4) 1171(19.4) 305(8.8)

Page 13/16 


\begin{tabular}{llll} 
Poorer & $774(17.6)$ & $495(19.2)$ & $279(14.9)$ \\
\hline Middle & $799(18.1)$ & $504(19.3)$ & $295(16.2)$ \\
\hline Richer & $837(20.0)$ & $491(20.5)$ & $346(19.2)$ \\
\hline Richest & $2515(28.9)$ & $1008(21.7)$ & $1435(40.8)$
\end{tabular}

Ever chewed chat

0.003

\begin{tabular}{ll} 
No & $6024(96.4)$ \\
Yes & $377(8.6)$ \\
\hline
\end{tabular}

Access to media

0.000

No

2898(49.5) 2063(56.7)

835(37.5)

Yes

3503(50.5) 1678(43.3)

1825(62.5)

Internet use

0.000

No

5606(92.5)

Yes

795(7.5)

Number of sexual partner (other than spouse)

0

1

Comprehensive HIV knowledge

No

Yes

Sexually Transmitted Infection in Past 12 months

\begin{tabular}{ll}
\hline No & $6376(99.8)$ \\
\hline Yes & $25(0.1)$ \\
\hline Ever tested for HIV & \\
\hline No & $3741(62.3)$ \\
\hline Yes & $2660(37.7)$ \\
\hline
\end{tabular}

Note: Frequency $(n)=$ unweighted count Percentage $(\%)=$ weighted percent

Table2. Bi-variable and Multivariable analysis on barriers to HIV testing among youth women Ethiopia 
Variables

Age

15-19

20-24

Residence

Urban

Rural

Marital status

Never union/single

Married

Divorce

Widowed

Living with partner

No longer living together
$\operatorname{cOR}(95 \% \mathrm{Cl})$

$\operatorname{AOR}(95 \% \mathrm{Cl})$

Ref

3.33(2.805-3.950) $\quad 2.18(1.800-2.652)$

\begin{tabular}{ll} 
Ref. & Ref \\
\hline $3.33(2.805-3.950)$ & $2.18(1.800-2.652)$
\end{tabular}




\begin{tabular}{lll}
0 & Ref & Ref. \\
\hline 1 & $1.64(1.216-3.870)$ & $2.48(1.350-4.551)$
\end{tabular}

\section{Comprehensive HIV knowledge}

$\begin{array}{lll}\text { No } & 0.51 & 0.68(0.530-0.861) \\ \text { Yes } & \text { Ref. } & \text { Ref. }\end{array}$

STIs in Past 12 months

\begin{tabular}{lll} 
No & Ref. & Ref. \\
\hline Yes & $2.31(1.530-4.108)$ & $1.50(0.379-5.906)$
\end{tabular}

